\title{
Modeling and Identification of Pneumatic Actuators
}

\author{
Yuval Tassa ${ }^{\dagger}$, Tingfan Wu*, Javier Movellan* \& Emanuel Todorov ${ }^{\dagger}$
}

\begin{abstract}
Pneumatic actuators are mechanically simple and robust, have good energetic properties due to air compressibility, and are relatively cheap. Despite these advantages they are difficult to control - pressure dynamics have typical timescales on the order of $100 \mathrm{~ms}$, and this delay can severely cripple simplistic control approaches. The solution is to use a modelbased controller with a good model of the pressure dynamics. Here we present a general parametric model of these dynamics based on both a theoretical analysis and an empirical study with a humanoid robot.
\end{abstract}

\section{INTRODUCTION AND RELATED WORK}

Pneumatic actuators are attractive for several reasons. They are naturally back-drivable, have low friction, tunable compliance and are very robust. They have a high strengthto-weight ratio - for example a typical cylinder of $5 \mathrm{~cm}$ diameter weighing $\sim 100$ grams, running at a standard 85 psi $(=590 \mathrm{KPa})$ above room pressure, produces 1160 Newtons or 260 pounds of force. Furthermore, the mechanical simplicity of pneumatics makes them inexpensive.

The central disadvantage or complication, is that they are much slower than electric motors or hydraulics, with dynamic timescales on the order of $\sim 100 \mathrm{~ms}$. In order to properly control a pneumatic system, a good model of these dynamics is required. Models of such systems can in general be classified as physical or parametric models. Physical models are constructed from first principles and attempt to conform as closely as possible to the underlying physical system. Parametric models are functions with unknown constants which are found using a curve-fitting procedure. While both types of models can have good predictive properties, the design objectives are different. The physical model attempts to accurately capture all the physical properties, regardless of how important they are for prediction. The design of a parametric model, while focusing on predictive power, must also take into account secondary objectives, like ensuring good convergence and eliminating local minima in the parameter space.

Previous work has focused either on precise physical models of pneumatic systems [1][2][3], or on linearized parametric models [4]. In this paper, we first develop a physical model from first principles, and then use this model to guide the design of a non-linear parametric model. The work most closely related to ours is [5], where quadratic polynomials are used as a basis for the non-linear parametrization. Rather than general polynomials, we use specially crafted functions, chosen to conform to the predictions of the initial physical

\footnotetext{
${ }^{\dagger}$ Computer Science and Engineering, University of Washington.

*Institute for Neural Computation, UC San Diego.
}

model. This paper is a natural continuation of our earlier work in [6].

\section{Physical Pneumatics Model}

\section{A. Ports, Valves and Chambers}

A pneumatic cylinder is a device with two chambers separated by a sliding bore. The air pressure in each chamber is controlled by valve which can connect the chamber to one of two ports: the supply port connects the chamber to a compressor and the exhaust port connects the chamber to room pressure. In some setups a single valve with two output

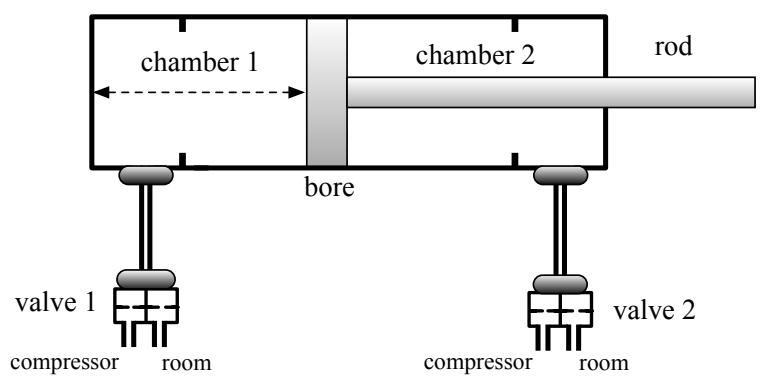

Fig. 1. Pneumatic cylinder. Schematics of a cylinder with two valves, one for each chamber. Each valve has two ports, one connected to room pressure $P_{r}$ and the other to the compressor $P_{c}$.

ports is connected to both chambers of a cylinder, allowing high pressure in either chamber, but not both. We chose the setup shown in Figure 1, where the chamber pressures can be controlled independently - to allow for the stiffness that results from high pressure on both sides. Another design choice was to use proportional valves rather than binary valves with a Pulse Width Modulation scheme. Proportional valves offer fine-grained control of the port size, and are also less noisy than a PWM setup. The details of our particular setup are further discussed in Section V, but the theoretical analysis should apply equally well to other configurations.

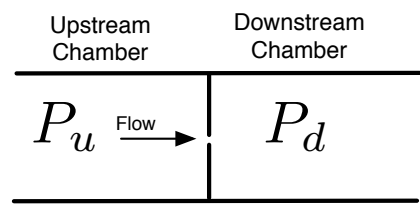

Fig. 2. Thin-plate port.

1) Port model: The port model describes the movement of fluid that occurs when connecting two chambers (upstream 
and downstream) of different pressure via a small orifice (Figure 2). Key assumptions are that the area of the port is small, that the plate separating the chambers is thin, that the fluid is a perfect gas, that the temperatures in the two chambers are equal, and that the flow is isentropic. Under these assumptions the mass flow $\dot{m}$ is the product of the orifice area $a$ and a function $\phi\left(p_{u}, p_{d}\right)$ of the upstream and downstream pressures:

$$
\dot{m}=a \cdot \phi\left(p_{u}, p_{d}\right)
$$

$\phi(\cdot, \cdot)$ is called the thin-plate flow function [7].

$$
\begin{gathered}
\phi\left(p_{u}, p_{d}\right)=\left\{\begin{array}{ll}
z\left(p_{u}, p_{d}\right) & \text { if } p_{u} \geq p_{d} \\
-z\left(p_{d}, p_{u}\right) & \text { if } p_{u}<p_{d}
\end{array} \quad\right. \text { (1b) } \\
z\left(p_{u}, p_{d}\right)= \begin{cases}\alpha p_{u} \sqrt{\left(\frac{p_{d}}{p_{u}}\right)^{\frac{2}{\kappa}}-\left(\frac{p_{d}}{p_{u}}\right)^{\frac{\kappa+1}{\kappa}}} & \text { for } p_{u} / p_{d} \leq \theta \\
\beta p_{u} & \text { for } p_{u} / p_{d}>\theta\end{cases}
\end{gathered}
$$

The physical constants $\kappa, \alpha, \beta$ and $\theta$ are described in the Appendix. Figure 3 shows the air-flow $\dot{m}$ as a function of the pressure in one of the chambers while the other chamber is at room pressure, for several orifice diameters. The function is continuously differentiable. When the upstream pressure is larger than $\theta$ times the downstream pressure, the flow becomes linear in the upstream pressure and independent of the downstream pressure.

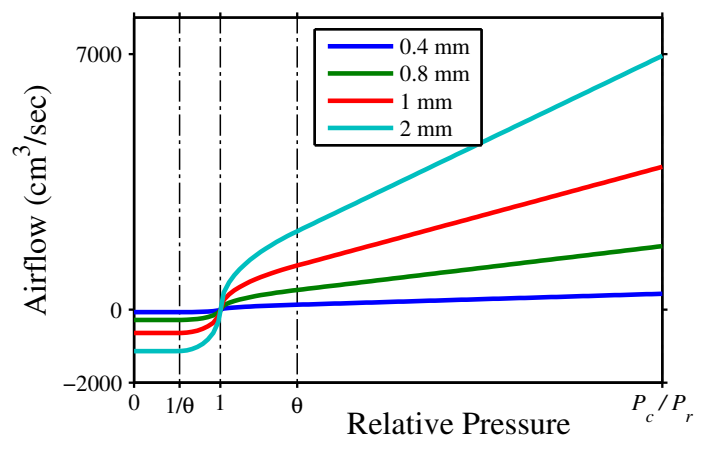

Fig. 3. Thin Plate Flow Function. We plot Eq. (1): the air flow $\dot{m}$ as a function of the ratio $p_{u} / p_{d}$. The downstream pressure $p_{d}$ is kept at room pressure $P_{r} \approx 100_{K P a}$, and $p_{u}$ is varied from 0 to the compressor pressure $P_{c} \approx 620_{K P a} \approx 90_{p s i}$. The 4 curves show the flow rate for 4 orifice diameters. The linear regime is outside the dotted vertical lines.

2) Two-port Chamber: The total flow of fluid mass into a chamber with 2 ports is the difference of the flows:

$$
\dot{m}\left(p, a_{c}, a_{r}\right)=a_{c} \phi\left(P_{c}, p\right)-a_{r} \phi\left(p, P_{r}\right)
$$

where $a_{c}, a_{r}$ are the orifice areas connecting the chamber to the compressor and room respectively, and $P_{c}, P_{r}$ are the respective constant pressures. Figure 4 shows this function to be monotonically decreasing, which corresponds to stable dynamics which converge to a steady-state pressure $p_{s s}$ given by $a_{c} \phi\left(P_{c}, p_{s s}\right)=a_{r} \phi\left(p_{s s}, P_{r}\right)$.

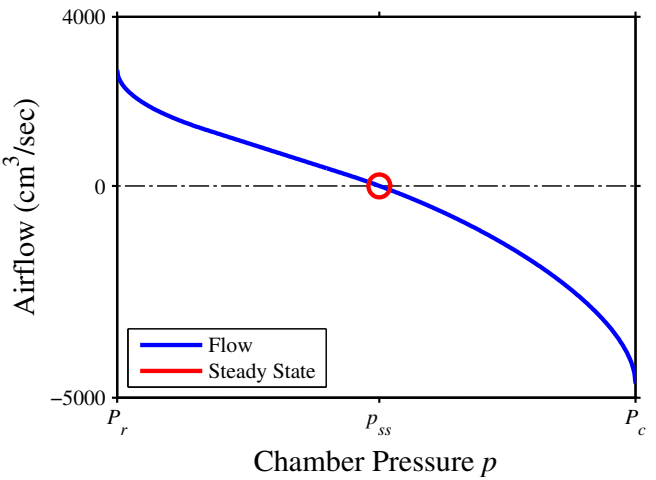

Fig. 4. Flow in a chamber with 2 ports. We plot Eq. (2): the mass flow $\dot{m}\left(p, a_{c}, a_{r}\right)$ for orifices of $1_{m m}$ radius: $a_{c}=a_{r}=\pi_{m m^{2}}$. The red circle shows the steady-state pressure which the dynamics converge to.

3) Valve model: A valve is a mechanism for controlling the orifice areas of the ports. Figure 5 illustrates a proportional valve. For an input voltage $u$, a moving part called the spool assumes a position which is linear in $u$, and partially or fully obstructs the ports. As the spool moves

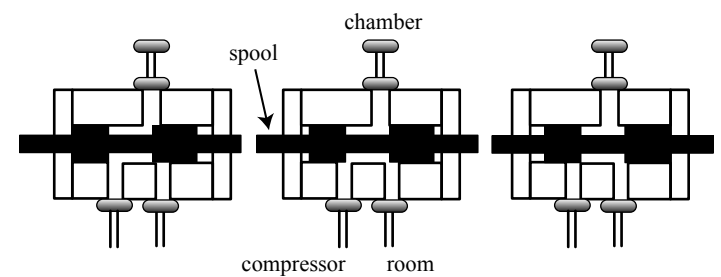

Fig. 5. Schematics of a proportional valve. A movable part called the spool (black) moves to block or unblock the ports. Left: exhaust port open, supply port closed. Center: exhaust and supply partially open. Right: exhaust closed, supply open.

over the port the effective area of the port will smoothly transition from a constant (very small) area when blocked, to a linearly increasing area when unblocked. The precise form of the transition depends on the relative shape of the port and the spool. We chose to model it with the function $\operatorname{smax}(x)=\left(\sqrt{x^{2}+1}+x\right) / 2$, which is a smooth approximation to $\max (x, 0)$.

$$
\begin{aligned}
& a_{c}(u)=L_{c}+\operatorname{smax}\left(\left(u-U_{c}\right) B-L_{c}\right) \\
& a_{r}(u)=L_{r}+\operatorname{smax}\left(\left(U_{r}-u\right) B-L_{r}\right)
\end{aligned}
$$

here $L_{c}, L_{r}$ are the respective minimal areas of the compressor and room port orifices, corresponding to leakage when the ports are sealed. $U_{c}, U_{r}$ are the voltage values at which the respective ports are sealed and $B$ is the coefficient which translates from voltage to area. Different relative sizes of the spool and the ports will lead to different partial obstructions at mid-voltage, as shown in Figure 6. 


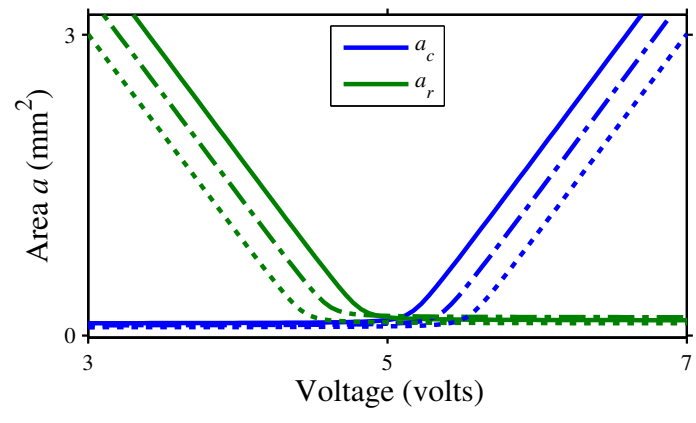

Fig. 6. Valve model. We plot Eq. (3): Areas of the compressor port $a_{c}$ and the room port $a_{r}$ as a function of the voltage $u$ applied to the valve. The different line-styles correspond to different choices of the parameters. The axes are scaled to the valves we use, see Section V.

4) Chamber Model: We can now write the pressure dynamics of a single chamber:

$$
\begin{aligned}
\dot{p}(p, u, \mathrm{v}, \dot{\mathrm{v}}) & =\kappa \frac{R T}{\mathrm{v}} \dot{m}-\kappa \frac{\dot{\mathrm{v}}}{\mathrm{v}} p \\
\dot{m}(p, u) & =a_{c}(u) \phi\left(P_{c}, p\right)-a_{r}(u) \phi\left(p, P_{r}\right)
\end{aligned}
$$

where $\mathrm{v}$ is the volume of the chamber, $\dot{\mathrm{v}}$ is the rate of change of that volume and $\kappa, R$ and $T$ are physical constants (see Appendix). The first term in the pressure dynamics equation (4a) is due to the flow from the valve, while the second one is due to compression from the piston.

5) Independence from the Mechanics: Given the volume $\mathrm{v}$ and its derivative $\dot{\mathrm{v}}$, the pressure dynamics are independent from the mechanical dynamics. Similarly, given the pressure difference between the two chambers, the force is known, and the mechanical system is independent of the pressure dynamics. Because the cylinders are rigidly attached to the limbs, $v$ is a deterministic function of the joint angles $\mathbf{q}$. Since barometers for measuring $p$ are cheap and accurate, as are potentiometers for measuring $\mathbf{q}$, we assume that both are indeed measured, and safely ignore the mechanics.

\section{PARAMETRIC Model Design}

The general parametric form of the pressure dynamics is

$$
\dot{p}=f(p, u, \mathrm{v}, \dot{\mathrm{v}} ; \mathbf{c}),
$$

where $\mathbf{c}$ is a vector of parameters to be fit to data measured from a real pneumatic system. In principle, equations $(1,3,4)$, constitute exactly such a model, but this model cannot be applied to a real system in its current form, for two reasons.

First, it contains parameters which are very difficult to measure or fit, e.g. the constants $B, U_{c}, L_{c}, U_{r}, L_{r}$ in the valve equation (3). These constants depend on the precise internal geometric alignment of the spool and the ports. The three line-styles in Figure 6 correspond to "educated guesses" of these constants.

Second, though air dynamics are notorious for their slowness, they can also be extremely fast. Consider the vertical scale of Figures $(3,4)$; it is $\sim 10^{4} \mathrm{~cm}^{3} / \mathrm{s}$. The chamber of a fully retracted cylinder can easily have a volume of $\sim 0.1 \mathrm{~cm}^{3}$, leading to a pressure change on a timescale of 10 microseconds. In practice the lower bound on the timescale is determined by the valve dynamics, but these are usually quite fast, on the order of $\sim 10 \mathrm{~ms}$ for proportional valves and much faster for switching valves. This means that in order to integrate (W.R.T time), we would need either a very small timestep or a variable-timestep integrator. This could be computationally expensive and would complicate differentiation (W.R.T state), which is often required for model-based control.

The model we want is cheap to compute and to differentiate, easy to integrate, and has parameters that can be fit reliably, using a simple procedure. The parametric form which satisfies all of these design requirements is

$$
\dot{p}=(s(u, \mathrm{v}, \dot{\mathrm{v}} ; \mathbf{c})-p) \cdot r(u, \mathrm{v}, \dot{\mathrm{v}} ; \mathbf{c}) .
$$

The function $s()$ has units of pressure and describes the steady-state pressure of the system; the function $r()>0$ has units of inverse time and describes the total change rate in the system. The central advantage of (6) over (5) is its linearity with respect to $p$. Assuming fixed values for $s$ and $r$ over a small time-step $h$, we can integrate (6) analytically:

$$
p(t+h)=s+(p(t)-s) e^{-r \cdot h}
$$

This integration scheme is stable for any time-step, and is easily differentiable if $s()$ and $r()$ are differentiable.

Although we do not use the physical model $(1,3,4)$ directly in our final identification scheme, we will use it to instruct our design of the parametric model (6) and its constituent functions $s(), r()$.

\section{A. Steady-State Pressure}

The steady-state pressure function $s()$ can be directly predicted from the physical model. Assuming a constant volume $\mathrm{v}$, we eliminate the second term of (4a) and numerically solve for the steady-state solution $\dot{m}(p)=0$. Figure 7 shows the

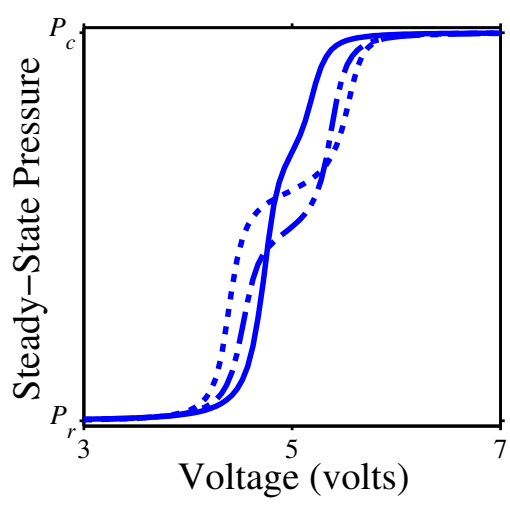

Fig. 7. Predicted steady-state pressure. $p_{s s}(u)$ : implicit solutions of $\dot{m}=0$. The three line-styles correspond to the three choices of valve model constants used in Figure 6. See Figure 10 for the same curves measured on the real system.

numerical roots of (4a) for different values of $u$. The effective function $p_{s s}(u)$ shown in the figure serves two purposes:

First, it shows us the required shape of the function $s(u, \cdot, \cdot ; \mathbf{c})$. It is a sigmoid with a flat kink at the origin. 
This flat region corresponds to the spool fully obstructing both ports. In this case the only flow is the leakage modeled by $L_{c}, L_{r}$ in (3), which is independent of the precise position of the spool. We used the quadratic sigmoid

$$
g(x)=x / \sqrt{x^{2}+1},
$$

and enabled the flat kink by adding a cubic term to the argument of the sigmoid (see below).

Second, it represents a theoretical prediction for the following simple experiment. By locking the cylinders in place we can fix the volume $\mathrm{v}$; changing the command voltage $u$ very slowly, so that the pressure is effectively at equilibrium, $\dot{m}$ vanishes and the measured pressure at the chamber should correspond to the plot in the figure.

\section{B. Rate}

The dependence of the rate function on the voltage $r(u, \cdot, \cdot ; \mathbf{c})$ roughly corresponds to the total port area $a_{c}(u)+$ $a_{r}(u)$, because $\dot{m}$ is linear in both $a_{c}$ and $a_{r}$. We therefore use the function $\sqrt{x^{2}+1}=\operatorname{smax}(x)+\operatorname{smax}(-x)$

$$
k(x ; a, b, c)=\sqrt{x^{2}+a^{2}}-a+b+c \cdot x,
$$

The parameters $a, b, c$ correspond to the size of the smooth area near the origin, a vertical shift of the whole function, and a tilt of the entire function around the origin, respectively. $a$ is related to the sealed regime of the valve, see below. The bias $b$ is required to model the leakage terms $L_{c}, L_{r}$. The tilt $c$ models asymmetries between the two ports.

\section{Volume Dependence}

Comparing (6) and (4a), we see that the volume v enters linearly in the denominator, and can therefore be confined to the denominator of $r()$. The volume velocity $\dot{\mathrm{v}}$ multiplies $p$ and linearly "drives" the ratio $r / s$, so it cannot be confined to one, but must appear in both $r()$ and $s()$.

\section{The Proposed Model}

Unlike the physical model where every term carries explicit units, the parametric model includes the necessary scaling and bias terms, which are found automatically by the fitting procedure. This means that sensors do not need to be calibrated independently. For example if the volume $\mathrm{v}$ is measured with a linear potentiometer on the piston (whose extension is linear with the volume), there is no need to explicitly convert to units of $\mathrm{cm}^{3}$. Similarly, the output voltage of the pressure sensor need not be calibrated into physical units and can remain in sensor units.

Putting the pieces together, our model has 9 free parameters $\mathbf{c}=\left(c_{1}, c_{2}, \ldots c_{9}\right)$ per valve-chamber system which must be determined. Additionally $c_{b}$ and $c_{s}$ are the bias and the gain of the steady-state pressure sigmoid and are computed explicitly as $c_{b}=\left(P_{c}+P_{r}\right) / 2$ and $c_{s}=\left(P_{c}-P_{r}\right) / 2$. One parameter $\left(c_{\gamma}\right)$ is chosen heuristically (see below). Written in sequential form, the model is given by $(8),(9),(10)$ :

$$
\begin{aligned}
\hat{u} & =u-c_{1} \\
\bar{g} & =g\left(c_{2} \hat{u}+c_{3} \hat{u}^{3}\right) \\
s & =c_{b}+c_{s} \bar{g}+c_{4} \dot{\mathrm{V}} \\
\bar{k} & =k\left(\hat{u}, c_{\gamma}, c_{9}, c_{8}\right) \\
r & =\frac{c_{7} \bar{k}+c_{5} \dot{\mathrm{v}}}{1+c_{6} \mathrm{v}} \\
\dot{p}(p, u, \mathrm{v}, \dot{\mathrm{v}} ; \mathbf{c}) & =(s-p) \times r
\end{aligned}
$$

More compactly, for $\hat{u}=u-c_{1}$ it is

$$
\begin{aligned}
\dot{p}=\left(c_{b}+\right. & \left.c_{s} \frac{c_{2} \hat{u}+c_{3} \hat{u}^{3}}{\sqrt{\left(c_{2} \hat{u}+c_{3} \hat{u}^{3}\right)^{2}+1}}+c_{4} \dot{\mathrm{v}}-p\right) \times \\
& \frac{c_{7}\left(\sqrt{\hat{u}^{2}+c_{\gamma}^{2}}-c_{\gamma}+c_{9}+c_{8} \cdot \hat{u}\right)+c_{5} \dot{\mathrm{v}}}{1+c_{6} \mathrm{v}} .
\end{aligned}
$$

Some of these parameters have physical interpretations that can lead to explicit constraints in the parameter space, see Table I. For example the "kink factor" $c_{3}$ must be larger than -1 to maintain the monotonicity of $s(u)$.

\begin{tabular}{|c|l|c|}
\hline parameter & \multicolumn{1}{|c|}{ interpretation } & comments \\
\hline \hline$c_{1}$ & valve-voltage origin, $\left(U_{c}+U_{r}\right) / 2$ & $2<c_{1}<8$ \\
\hline$c_{2}$ & valve-voltage scale, $B$ & $-1<c_{3}$ \\
\hline$c_{3}$ & kink factor & \\
\hline$c_{4}$ & $\partial s / \partial \dot{\mathrm{v}}$ & \\
\hline$c_{5}$ & $\partial r / \partial \dot{\mathrm{v}}$ & \\
\hline$c_{6}$ & volume-sensor scale & $-1<c_{8}<1$ \\
\hline$c_{7}$ & $B$ of Eq. $(3)$ & $0<c_{9}$ \\
\hline$c_{8}$ & rate asymmetry & $=\left(P_{c}+P_{r}\right) / 2$ \\
\hline$c_{9}$ & leakage, $\left(L_{c}+L_{r}\right) / 2$ & $=\left(P_{c}-P_{r}\right) / 2$ \\
\hline \hline$c_{b}$ & pressure-sensor bias & see below \\
\hline$c_{s}$ & pressure-sensor scale & \\
\hline$c_{\gamma}$ & voltage range of leaky regime & \\
\hline
\end{tabular}

TABLE I

INTERPRETATION OF MODEL PARAMETERS

The parameter $c_{\gamma}$ gives the range of voltage around $u=$ $c_{1}$ for which the ports are "almost" sealed, i.e. when the leakage flow is not small relative to the total flow. In terms of Eq. (3) this corresponds to $U_{r}<u<U_{c}$. Because $c_{\gamma}=$ 0 creates a non-differentiable point, it is important that it remain positive, yet at $c_{\gamma}=0$ the derivative $\partial k / \partial c_{\gamma}$ also vanishes, which leads to a bad local minimum for the fitting procedure. For these reasons we set it globally to $c_{\gamma}=0.1_{V}$.

The constant 1 in the denominator of $(10 \mathrm{e})$ is required to collapse the multiplicative invariance of $\left(c_{5}, c_{6}, c_{7}\right)$, while still allowing all 3 degrees of freedom. It can also be understood as the bias of the volume sensor, in units of volume sensor bias.

\section{EXPERIMENT}

We identified the humanoid robot shown in Figure 8, made by Kokoro in Japan. It has 44 pneumatically-actuated dofs; 6 of them are in the hands which were removed for the 
purposes of this paper, so here we are controlling only 38 dofs. Our long-term goal is to be able to make this humanoid perform various life-like movements using model based control. Before attempting such a challenging task, however, we need to identify the pneumatic system. Initial test were performed and reported in [6], where we experimented with a 2-dof arm made by the same manufacturer using the same components.

Each joint is driven by either a linear or a rotary pneumatic cylinder. The drive is direct, without any gears, belts or cables (except for a couple of joints in the humanoid). This makes the system both more compliant and more robust - indeed the robot has been hitting its joint limits at high speeds during the identification tests, without any damage. Each cylinder has two chambers fitted with solid-state pressure sensors. Each chamber is connected to a proportional valve, which can be open towards the compressor (at 90 psi) or towards the room (where the atmospheric pressure is 14.7 psi) or it can be sealed (up to small leakage). The joint angles are measured by potentiometers. The humanoid has 114 sensors and 76 controls.
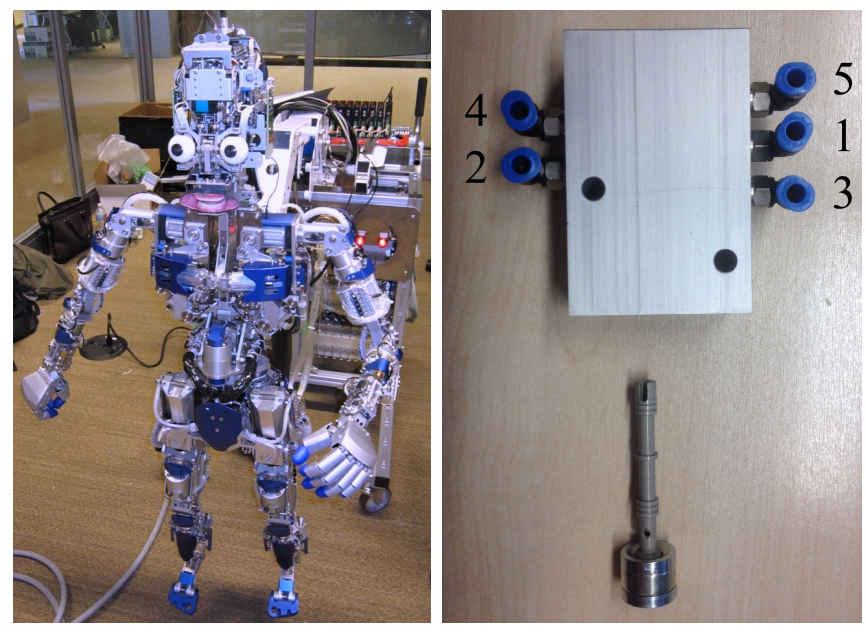

Fig. 8. Left: Pneumatic humanoid robot. Right: Valve and spool. In the classic setup the compressor is connected to 1 , the chambers of a single piston are connected to 4 and 2 , and 5 and 3 are respective exhaust ports. We connect a single chamber to 4 with an exhaust at 5 , and plug ports 2 and 3 .

We use the MPYE proportional directional control valves by FESTO. The valves take a command voltage $0<u<10$ Volts, but we used $3<u<7$ Volts to avoid hitting the limits inside the valve. These valves are of the so called "5/3" type, which are usually connected to both chambers of a cylinder, and can alternatingly conduct the compressor pressure either to one chamber or to the other, but not to both. We are interested in exploiting the stiffness control that is made possible by pressurizing both chambers, so chose to connect two valves to each cylinder, one to each chamber. In order to apply our model to the standard setup, the control voltage data for the single valve would simply be duplicated, and each chamber identified separately.

We use National Instruments I/O boards. The valves have
$100 \mathrm{~Hz}$ bandwidth, thus the control loop runs at $100 \mathrm{~Hz}$. The pressure sensors and potentiometers are analog devices that can be sampled at arbitrary rates. We are sampling all sensors at $20 \mathrm{KHz}$ and average every 200 samples, for a $100 \mathrm{~Hz}$ sensorimotor loop. Such averaging is beneficial because the sensor noise is essentially white. The software system consists of the NIDAQmx drivers and a $\mathrm{C}$ function which reads the driver buffers, returns averaged sensor data, and sets the desired valve voltages. Data analysis was done in MATLAB.

\section{A. Flow}

We measured the steady-state flow rate as a function of voltage for the two ports in the standard setup for a single valve using a FESTO SFAB-200U flow sensor, see Figure 9. Since these are proportional to port area it can be seen that our valve model in (Figure 6) captures the principal features of the area function.

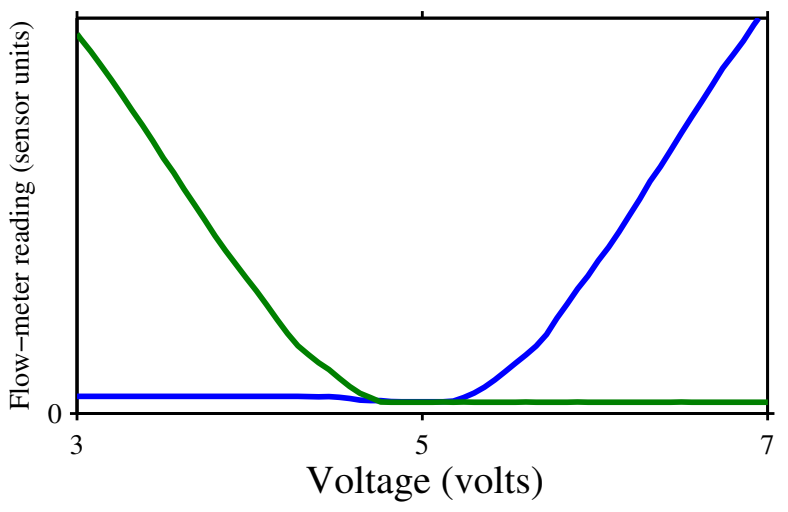

Fig. 9. Measured valve flow. Compare to Figure 6.

\section{B. Steady-State Pressure}

We performed the experiment described in Section III-A, and measured the chamber pressure for a locked cylinder, while changing the command voltage $u$ very slowly, to measure the steady-state pressure function $s()$. We performed this measurement for all 78 valve-chamber pairs (Figure 10), and noted that the resulting curves compared favorably to the solutions predicted by the physical model (Figure 7).

\section{Identification}

We fit the parameters $c_{1 \ldots 9}$ using standard nonlinear leastsquares on measured data. We recorded $100 \mathrm{~s}\left(10^{5}\right.$ data points) of controlled movement during which the robot was flailing its limbs (see video attachment). These movements were controlled by a simple PID controller W.R.T the potentiometer reading, and were designed to explore the statespace as violently as possible, without hitting any joint limits so strongly that the robot would break. For each of the 76 valve-chamber systems, the data consisted of the voltage $u_{t}$, chamber pressure $p_{t}$, and joint-potentiometer reading $\mathrm{v}_{\mathrm{t}}$ (which are proportional to the volume). The latter were finite-differenced to obtain joint velocities $\dot{\mathrm{v}}_{t}$. Rather than fit 


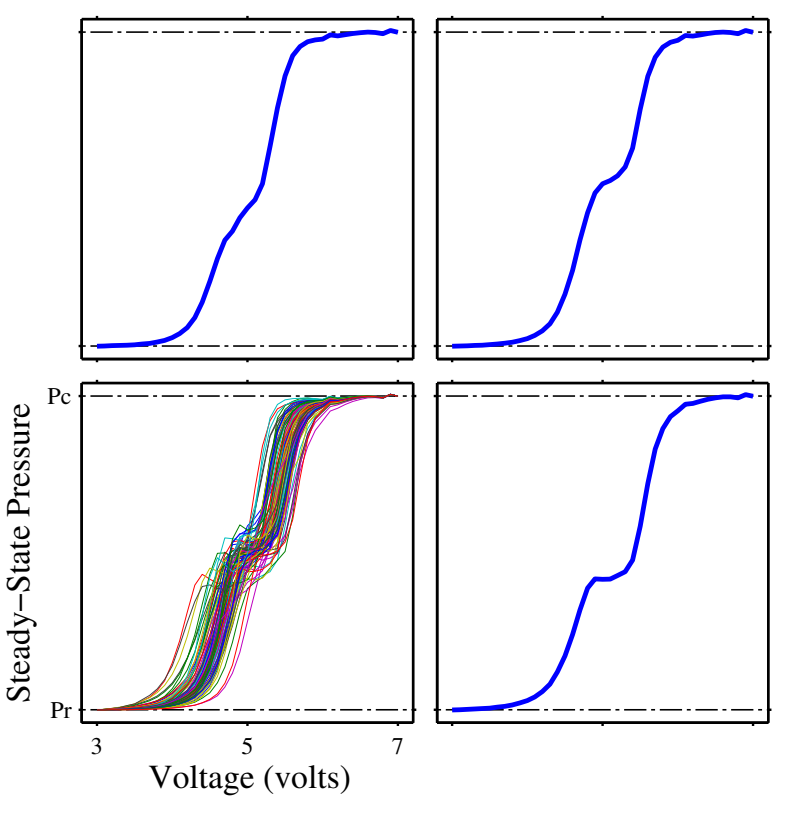

Fig. 10. Measured steady-state pressure. Bottom left: overlay of steadystate pressure measurement for all 76 valves. Above and to the right: Three typical curves. Compare to Figure 7, which shows curves predicted by the physical model.

$\dot{p}$ directly, we used the integration formula (7) and fit the pressure at the next timestep.

$$
\mathbf{c}^{*}=\underset{\mathbf{c}}{\operatorname{argmin}} \sum_{t}\left(p_{t+h}-\hat{p}_{t+h}\left(p_{t}, u_{t}, \mathrm{v}_{t}, \dot{\mathrm{v}}_{t} ; \mathbf{c}\right)\right)^{2}
$$

where $\hat{p}_{t+1}$ is the value predicted by (7). The values of $\mathbf{c}$ were constrained by the limits in Table I. In our tests the optimization always converged to the same minimum, from different initial conditions. We have no formal guarantee of convexity, but we had never encountered bad local minima with this parametrization. It is worth noting that the particular functional form we use here is the result of a long test process, where many other functional forms were tried, which often misconverged to bad minima. The empirically good convergence properties of our model, though difficult to quantify, are one of its strongest features.

Figure 11 shows an overlay of the predicted and measured change in pressure for a single valve-chamber system. Clearly the main features of the pressure derivative function are captured by the model, but a simple overlay does not properly quantify the quality of the fit.

\section{Multi-step Prediction}

Our end goal is to use this model for model-based predictive control. Since we do not yet have a a full kinematic and dynamic model of our robot, we can do the next-best thing, which is to test how good the prediction is for a multistep horizon. In this scenario we have a measurement of the current pressure $p_{t}$, and a planned sequence of command voltages $u_{t \ldots T}$. Given a prediction of the joint angles $\mathbf{q}_{t \ldots T}$ (and therefore of future chamber volumes
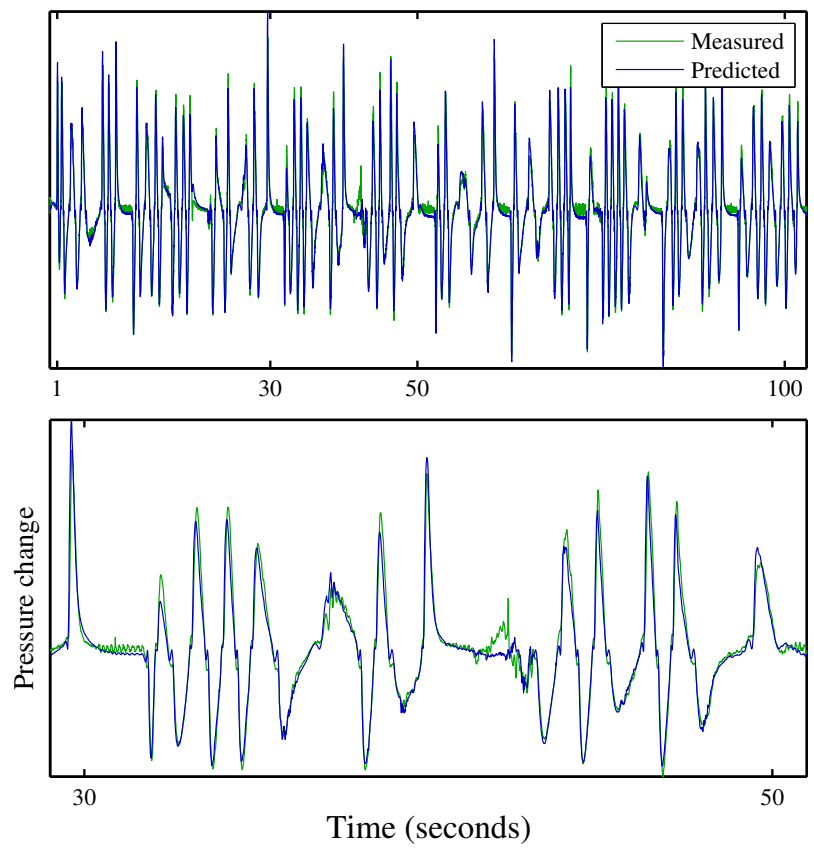

Fig. 11. Pressure change fit. Shown for a a typical valve-chamber system The light curve shows the measured pressure difference $p_{t+h}-p_{t}$, the dark curve shows the predicted change $\hat{p}_{t+h}-p_{t}$. The bottom figure shows the same data at higher temporal resolution.

and velocities $\mathrm{v}_{t \ldots T}, \dot{\mathrm{v}}_{t \ldots T}$ ), we can recursively integrate the pressure dynamics. Of course future joint angles depend on the chamber pressures, so this prediction would be performed simultaneously for $p$ and q. Here however, we can simply take the measured values of $u, \mathrm{v}, \dot{\mathrm{v}}$, while integrating $p$ using

$$
\begin{aligned}
\hat{p}_{0} & =p_{0} \\
\hat{p}_{t+h} & =\hat{p}_{t+h}\left(\hat{p}_{t}, u_{t}, \mathrm{v}_{t}, \dot{\mathrm{v}}_{t} ; \mathbf{c}\right) .
\end{aligned}
$$

Thus, the predicted pressure $\hat{p}_{t}$ is allowed to diverge from the measured pressure $p_{t}$. Figure 12 shows this divergence for the same valve-chamber system of Figure 11. $\hat{p}_{t}$ was initialized with $p_{t}$ at the dotted grid lines and integrated thereafter. Surprisingly, almost no drift was detected.

Figure 13 shows the results of the same test performed for all 76 valve-chamber systems. Starting from 48 time points in each dataset, we integrated the pressure prediction for 2 seconds. We plot the standard deviation of the prediction error as a function of time for each valve separately, and for all $3648=76 \times 48$ integration sequences. Note that the vertical axis is scaled by $\left(P_{c}-P_{r}\right) / 10$, so the expected drift of the predicted pressure is less than $\% 10$ of the difference between chamber and room pressure, and usually less than $\% 4$. We attribute the surprising lack of drift both to the quality of the fit and to the parametrization (5), which correctly captures the dynamical convergence to a set-point exhibited by the pressure. 

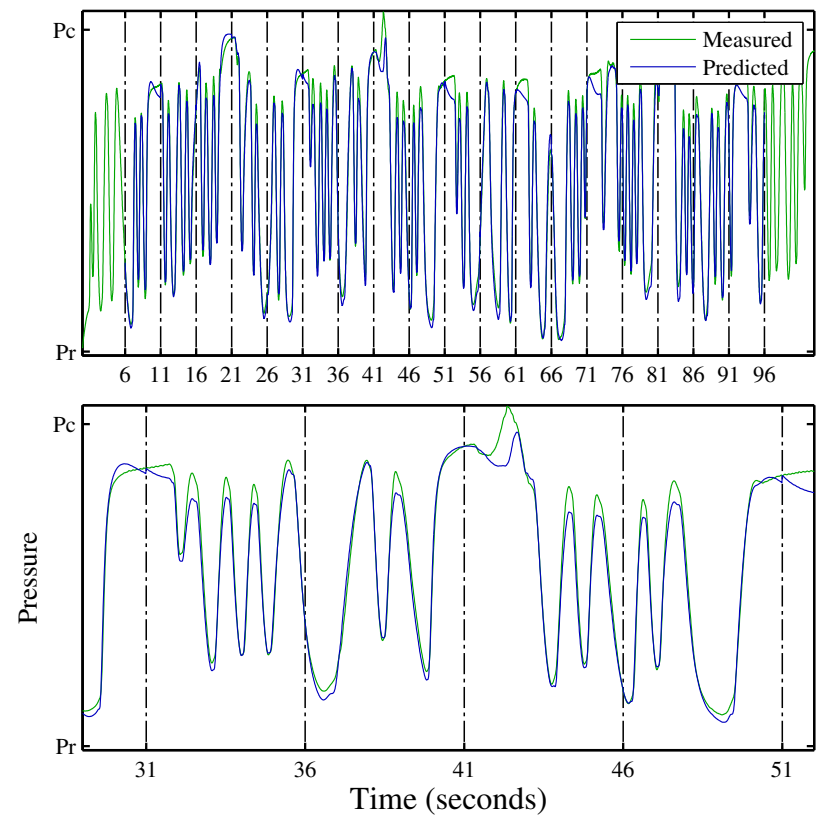

Fig. 12. Pressure prediction. The light curve shows the actual pressure $p_{t}$, the dark curve shows the integrated pressure, starting at the dotted grid lines. The bottom figure shows the same data at higher temporal resolution.

\section{APPENDIX}

The physical constants in Eq. (1) are given by:

$$
\begin{aligned}
\alpha & =C \sqrt{\frac{2 M}{Z R T} \frac{\kappa}{\kappa-1}} \\
\beta & =C \sqrt{\frac{\kappa M}{Z R T}\left(\frac{2}{\kappa+1}\right)^{\frac{\kappa+1}{\kappa-1}}} \\
\theta & =\left(\frac{\kappa+1}{2}\right)^{\frac{\kappa}{\kappa-1}}
\end{aligned}
$$

\section{$M, Z, R, T, \kappa, C$ are defined in Table II.}

\begin{tabular}{|l|l|l|}
\hline Gas Molecular Mass & $M$ & 0.029 for air, $\mathrm{Kg} / \mathrm{mol}$ \\
\hline Temperature & $T$ & $K^{\circ}$ \\
\hline Universal Gas Constant & $R$ & $8.31\left(\mathrm{~Pa} \cdot \mathrm{m}^{3}\right) /\left(\mathrm{mol} \mathrm{K}^{\circ}\right)$ \\
\hline Discharge coefficient & $C$ & 0.72, dimensionless \\
\hline Compressibility Factor & $Z$ & 0.99 for air, dimensionless \\
\hline Specific Heat Ratio & $\kappa$ & 1.4 for air, dimensionless \\
\hline Mass Flow & $\dot{m}$ & $\mathrm{Kg} / \mathrm{s}$ \\
\hline Pressure & $p$ & Pascals \\
\hline Area & $a$ & $\mathrm{~m}^{2}$ \\
\hline
\end{tabular}

TABLE II

PARAMETERS AND UNITS OF THE THIN-PLATE PORT MODEL.

\section{ACKNOWLEDGMENT}

This work was supported by the National Science Foundation.

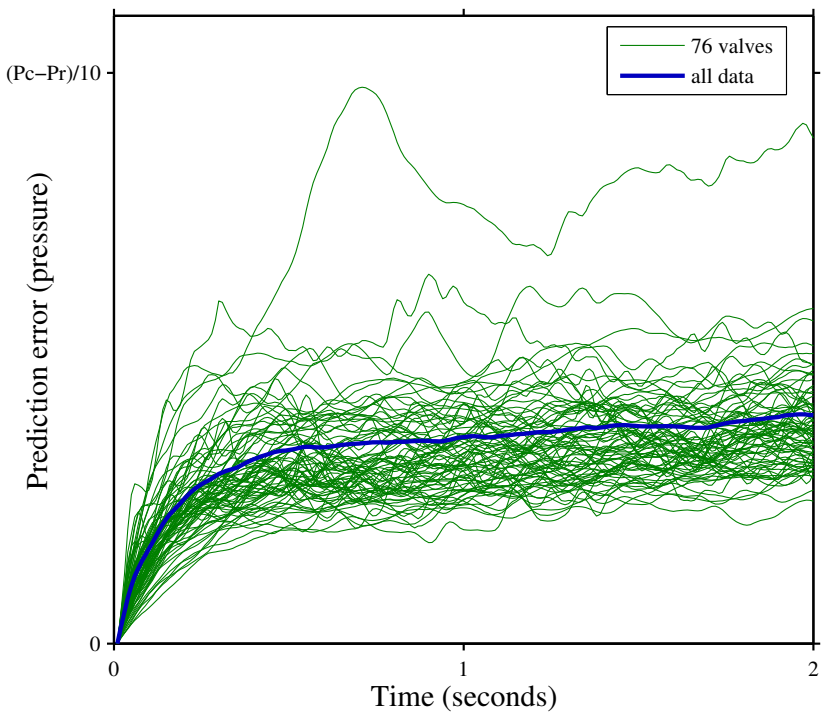

Fig. 13. Drift of pressure prediction. Expected deviation of the pressure prediction from true pressure as a function of time from the last accurate measurement. Note that the vertical scale is $\% 10$ of the pressure difference $P_{c}-P_{r}$.

\section{REFERENCES}

[1] J. Bobrow and B. McDonell, "Modeling, identification, and control of a pneumatically actuated, force controllable robot," IEEE Transactions on Robotics and Automation, vol. 14, no. 5, pp. 732 -742, Oct. 1998.

[2] N. Gulati and E. Barth, "Non-linear pressure observer design for pneumatic actuators," in Proceedings, 2005 IEEE/ASME International Conference on Advanced Intelligent Mechatronics, July 2005, pp. 783 -788 .

[3] — - "A globally stable, load-independent pressure observer for the servo control of pneumatic actuators," IEEE/ASME Transactions on Mechatronics, vol. 14, no. 3, pp. 295 -306, June 2009.

[4] S. R. Pandian, F. Takemura, Y. Hayakawa, and S. Kawamura, "Pressure observer-controller design for pneumatic cylinder actuators," IEEE/ASME Transactions on Mechatronics, vol. 7, no. 4, pp. 490 499, Dec. 2002.

[5] Z. Rao and G. Bone, "Nonlinear modeling and control of servo pneumatic actuators," IEEE Transactions on Control Systems Technology, vol. 16, no. 3, pp. $562-569$, May 2008.

[6] E. Todorov, C. Hu, A. Simpkins, and J. Movellan, "Identification and control of a pneumatic robot," in Biomedical Robotics and Biomechatronics (BioRob), 2010 3rd IEEE RAS and EMBS International Conference on, 2010, p. 373-380.

[7] R. H. Perry, D. W. Green, and J. O. Maloney, Perry's chemical engineers' handbook. McGraw-Hill New York, 1984, vol. 7. 